\title{
Lymphocyte-Depleted Classic Hodgkin Lymphoma by Ann Arbor Stage
}

National Cancer Institute

\section{Source}

National Cancer Institute. Lymphocyte-Depleted Classic Hodgkin Lymphoma by Ann

Arbor Stage. NCI Thesaurus. Code C141222.

An anatomic stage for lymphocyte-depleted classic Hodgkin lymphoma based on the Ann Arbor classification criteria. 